\title{
Zur Synonymie von Litosiphon pusillus auf der Basis von Kulturversuchen
}

\author{
P. Kornmann \& P.-H. Sahling \\ Biologische Anstalt Helgoland (Meeresstation); D-2192 Helgoland, \\ Bundesrepublik Deutschland
}

\begin{abstract}
The synonymy of Litosiphon pusillus on the basis of culture studies. In June 1988, $L$. pusillus was collected for the first time in the Helgoland area. On the basis of observations in cultures, Pedersen's hypothesis that the microthalli of $L$. pusillus are identical with those of three Pilocladus species is discussed. The general morphology as well as the shape and dimensions of the unilocular sporangia are clearly different in Pilocladus and Litosiphon microthalli. Therefore, Pedersen's opinion cannot be accepted.
\end{abstract}

\section{EINLEITUNG}

Litosiphon pusillus (Carmich. ex Hook.) Harvey wurde Ende Juni 1988 erstmalig im Helgoländer Yachthafen epiphytisch auf Laminaria gefunden. Die untersuchten Fäden enthielten entweder nur plurilokuläre oder nur unilokuläre Sporangien.

Die Alge war schon mehrfach Gegenstand eingehendér Studien. Sauvageaus Beobachtungen (1933) enthalten neben einigen schwer einzuordnenden Einzelheiten wesentliche Ergebnisse. L. pusillus ist eine Eclipsiophycee. Die Epiphyten auf Saccorhiza tragen im Frühjahr plurilokuläre Sporangien, im Sommer und Herbst kommen unilokuläre hinzu. In Sauvageaus Kulturen vom März 1932 entstanden auf einem wenigzelligen Protonema Pflanzen mit plurilokulären Sporangien. In den Versuchen vom Juni 1928 waren die Protonemen größer; sie bildeten zunächst auch aufrechte Fäden, später aber einreihige pluri- und birnförmige unilokuläre Sporangien auf den kriechenden Fäden. Aus Fäden mit unilokulären Sporangien erhielt Sauvageau Mikrothalli mit langen einreihigen plurilokulären Sporangien, sie wurden als die überwinternde Generation angesehen.

Untersuchungen von Kylin (1933) und Dangeard (1965) brachten keine weiteren Erkenntnisse. Beide Autoren - in der Vorstellung eines antithetischen Generationswechsels befangen - glaubten ihre Beobachtungen in diesem Sinne deuten zu können. Nygren $(1975,1979)$ hat die entwicklungsgeschichtlichen Befunde Sauvageaus bestätigt und nachgewiesen, daß die heteromorphen Generationen von $L$. pusillus dieselbe Kernphase haben, daß also im unilokulären Sporangium keine Reduktionsteilung erfolgt.

Nach diesen Feststellungen wäre eigentlich eine nochmalige Untersuchung überflüssig erschienen, wenn nicht die Mikrothalli von L. pusillus durch Pedersen (1984) eine ungewöhnliche Interpretation erfahren hätten. Er identifizierte sie mit Arten der Gattun- 
gen Pilocladus und Streblonema, die damit Synonyme von Litosiphon pusillus wären. Unsere Kulturversuche sollten eine Stellungnahme zu dieser Hypothese ermöglichen.

\section{MATERIAL UND METHODE}

Für die Ausgangskulturen wurde Schreiberlösung mit einem Zusatz von $\mathrm{GeO}_{2}$ benutzt. Je ein Fadenstück mit pluri- bzw. unilokulären Sporangien lieferte an drei aufeinanderfolgenden Tagen Schwärmer. Beide Versuchsreihen enthielten je eine Deckglaskultur, bei der ein Deckglas mit dem Fadenstück in einem Tropfen Nährlösung auf der Flüssigkeitsoberfläche der Kulturschale schwamm. Nachdem sich die Schwärmer in dem Tropfen festgesetzt hatten, wurden die Deckgläser untergetaucht. In den Parallelkulturen lag das jeweilige Fadenstück einen Tag lang auf dem Boden der Plastikschalen, wo sich die Schwärmer festsetzten.

Nach 4 bis 5 Tagen wurden die Deckgläser zerteilt. Hierdurch standen gleichartige Inokulate für die Weiterkultur zur Verfügung. Die Deckglasfragmente wurden in die in unseren Kulturversuchen bewährte Provasoli-Lösung mit einem Zusatz von Erdabkochung übertragen. Einheitliche Licht- und Temperaturbedingungen - 14stündige Beleuchtung mit etwa 1000 bis $1700 \mathrm{Lux}, 14-15^{\circ} \mathrm{C}$ Raumtemperatur - genügten, um alle Entwicklungsstadien von Litosiphon pusillus in erstaunlich kurzer Zeit zu erhalten. Es bestand daher kein Anlaß, die Versuchsbedingungen zu variieren.

\section{KULTUREN MIT PLURI-ZOOSPOREN DES AUSGANGSFADENS}

Im Gegensatz zur Entwicklung, die Sauvageau (1933) und Kylin (1933) beschrieben hatten, und die auch wir später erhielten (vgl. Fig. 20-21), waren die 4 Tage alten Keimlinge unregelmäßig gewundene Fädchen; nur auf einigen erhob sich ein deutlich erkennbarer SproB (Fig. 2). Zu diesem Zeitpunkt wurde ein Deckglassplitter in eine Schale mit Provasoli-Medium umgesetzt. In der Ausgangskultur mit Schreiberlösung hatten sich nach sieben Tagen knotige aufrechte Sprosse oder auch plurilokuläre Sporangien entwickelt (Fig. 3), in der Parallelkultur mit Provasoli-Medium waren dagegen nach vier Tagen kräftige aufrechte Fäden entstanden (Fig. 4, 5). Die Figuren 6 und 7 zeigen die entsprechenden Kulturen im Alter von 10 Tagen bei gleicher Vergrößerung.

Dieses Experiment konnte mit den übrigen Deckglassplittern in Abständen von 1 bis 2 Wochen und zuletzt nach knapp 5 Monaten mit dem gleichen Erfolg wiederholt werden. Trotz ihres kümmerlichen Wachstums blieben die Pflänzchen in der Schreiberlösung lebensfähig, und es wurden unter entsprechenden Kulturbedingungen überraschend schnell normale Thalli gebildet. Es liegt nahe, dieses Kulturergebnis im Sinne der Fähigkeit überwinternder Stadien zu einer raschen Entwicklung bei Verbesserung der Lebensbedingungen zu bewerten.

In der 13 Tage alten Kultur hatten einige Fäden schon plurilokuläre Sporangien entleert (Fig. 8), zugleich war das basale System mit einreihigen plurilokulären Sporangien besetzt (Fig. 9). Fig. 10 zeigt den Bewuchs auf dem Schalenboden der 21 Tage alten Kultur: aus den dicken Zoosporen der Fadensporangien sind Fäden entstanden, aus den Zoosporen der einreihigen Sporangien Mikrothalli. Durch die große Zahl der rasch sich entwickelnden Schwärmer und ihre kurze Vegetationszeit wurden solche Kulturen schnell von Nachkommenschaften der Mikrothalli überwuchert und wegen Nährstoffmangels bald überständig. 


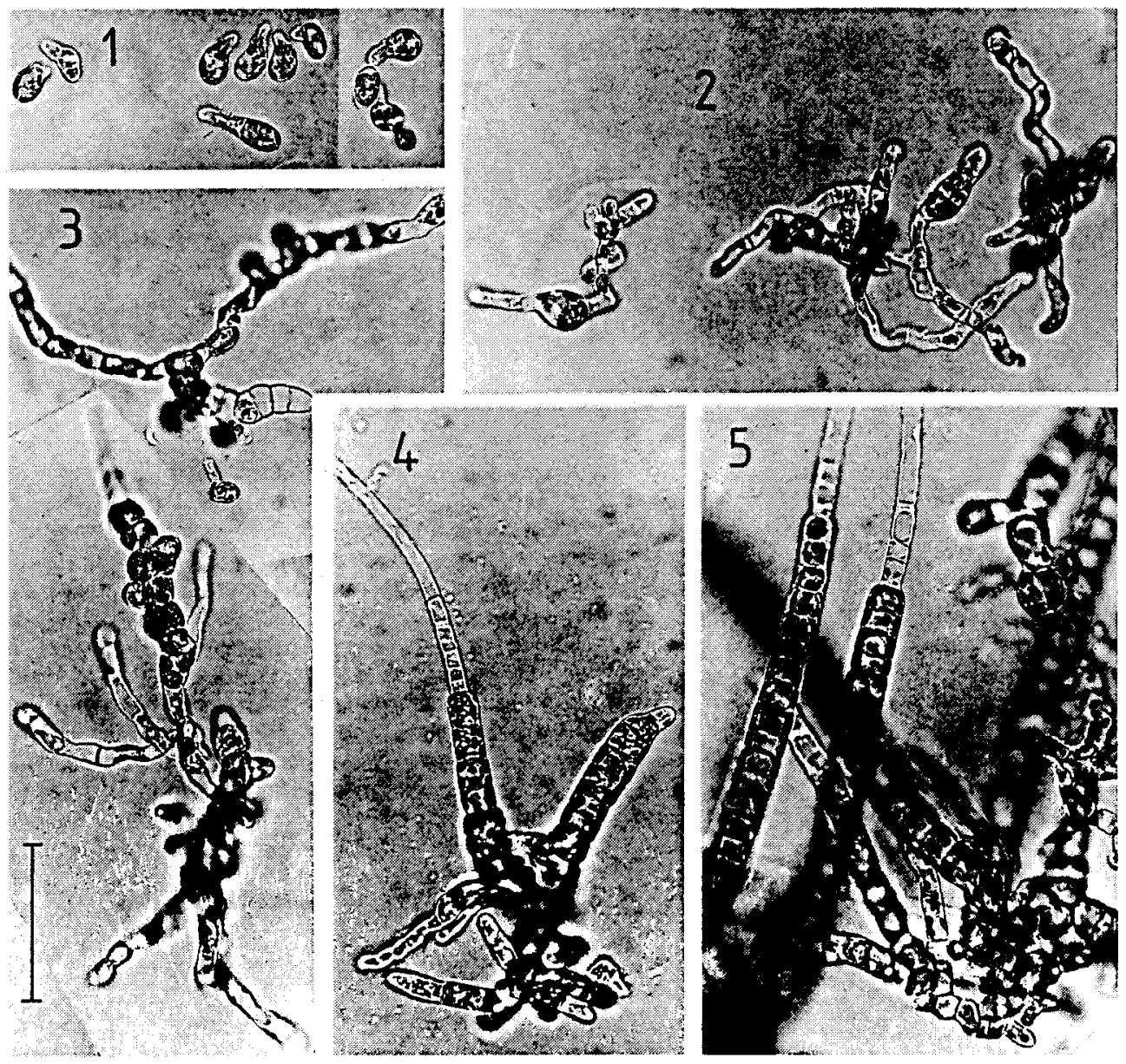

Fig. 1-5. Litosiphon pusillus, aus Schwärmern plurilokulärer Sporangien des Naturmaterials. Fig. 1-3: Keimlinge in Schreiber-Medium auf dem am 4. Tage zerteilten Deckglas, 1, 4 bzw. 8 Tage alt. Fig. 4 und 5: auf einem am 4. Tage in Provasoli-Medium umgesetzten Deckglassplitter, 6 bzw. 8 Tage alt. Maßstrecke $=50 \mu \mathrm{m}$

\section{KULTUREN MIT UNI-SCHWÄRMERN DES AUSGANGSMATERIALS}

Die Figuren 11 und 12 zeigen eine 5 Tage alte Kultur aus Schwärmern der unilokulären Sporangien des Ausgangsmaterials in Schreiber-Medium. An diesem Tage wurde das Deckglas zerbrochen und die Splitter in Provasoli-Medium übertragen. Schon 7 Tage später waren auf den niederliegenden Fäden zahlreiche plurilokuläre Sporangien und einzelne aufrechte Fäden entstanden (Fig. 13, 14). Die Figuren 17 und 18 vergleichen Ausschnitte aus der ursprünglichen und der erneuerten Kultur im Alter von 16 Tagen miteinander. Während der 11 Tage, die zwischen den Figuren 11 und 17 liegen, sind die fädigen Pflänzchen nur wenig größer geworden, dagegen haben sie sich in der ProvasoliNährlösung zu stark verzweigten Mikrothalli entwickelt, denen zum Teil kräftige auf- 

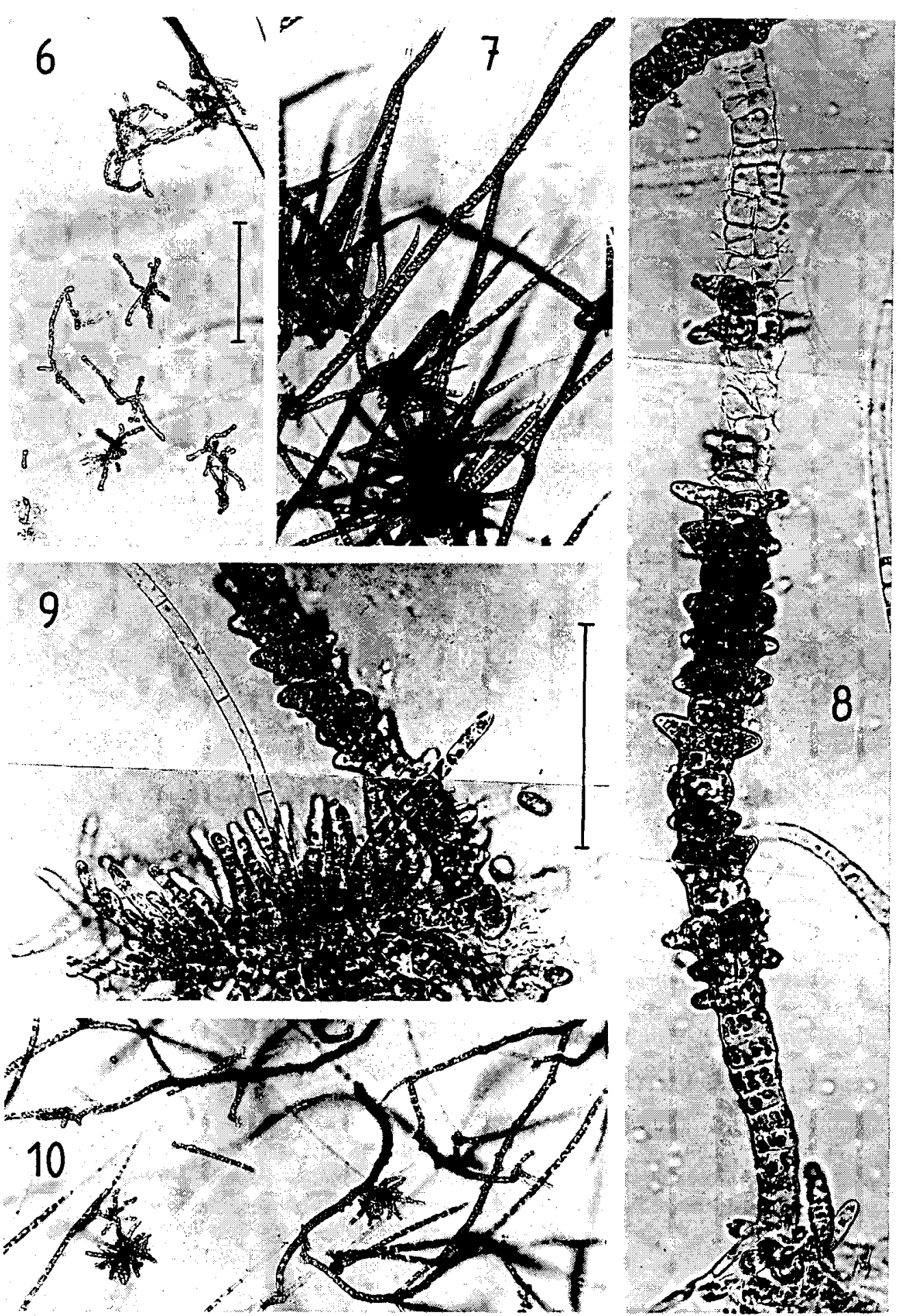

Fig. 6-10. Litosiphon pusillus, Fortsetzung. Fig. 6 und 7: Pflänzchen auf einem ursprünglichen und einem umgesetzten Deckglassplitter, 10 Tage alt. Fig. 8: Faden mit großen plurilokulären Sporangien, 13 Tage alt. Fig. 9: Basallager mit einreihigen plurilokulären Sporangien, 13 Tage alt. Fig. 10: Schalenboden der 21 Tage alten Kultur mit jungen Makro- und Mikrothalli. Maßstrecken: Fig. 6, 7 und $10=200 \mu \mathrm{m}$; Fig. 8 und $9=100 \mu \mathrm{m}$ 

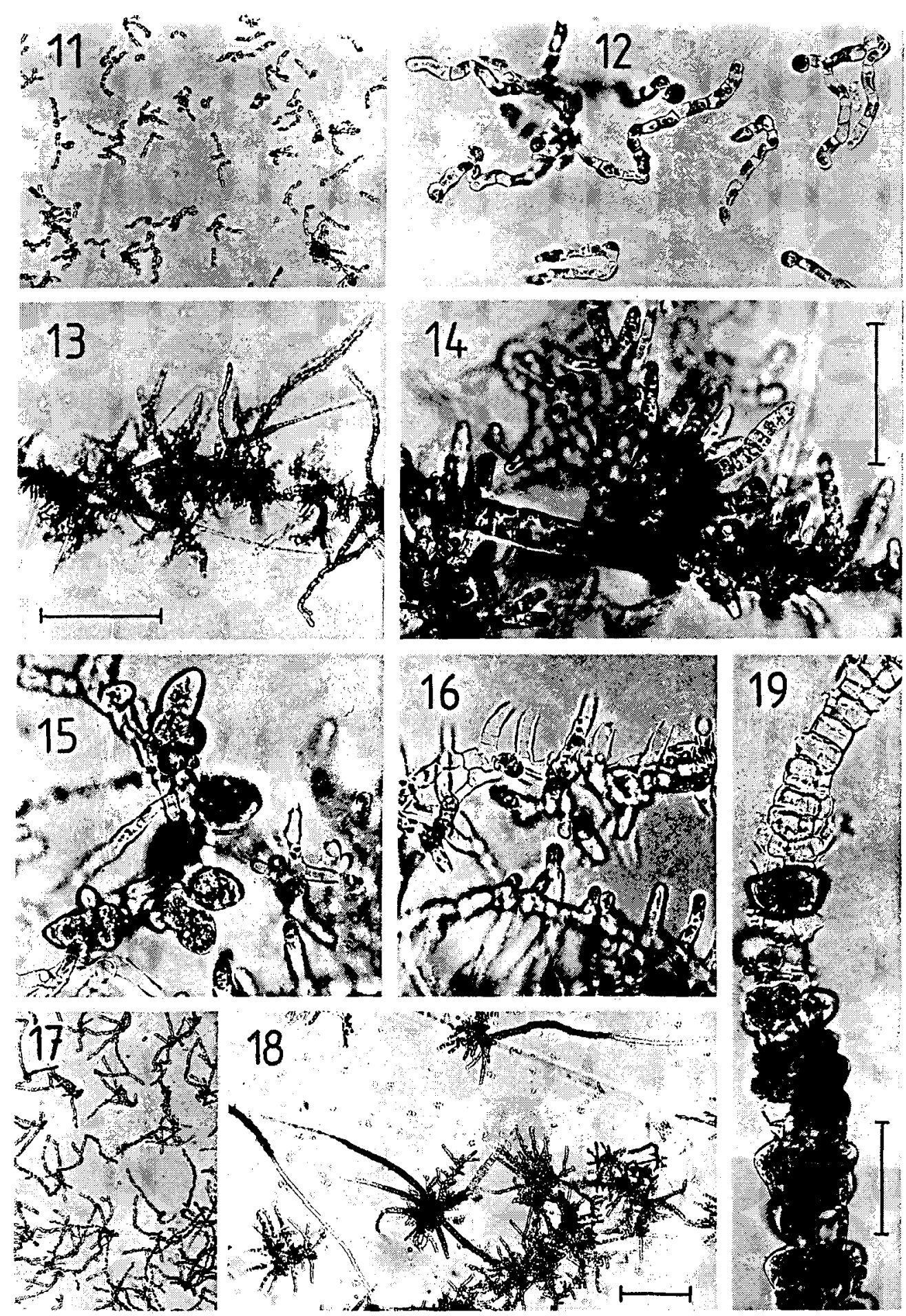

Fig. 11-19. Litosiphon pusillus, aus Schwärmern unilokulärer Sporangien des Naturmaterials. Fig. 11 und 12: Keimlinge auf dem noch nicht zerteilten Deckglas, 5 Tage alt. Fig. 13 und 14: auf einem am 5. Tage umgesetzten Deckglassplitter, 12 Tage alt. Fig. 15 und 16: wie die vorigen, 15 Tage alt. Fig. 17 und 18: Deckglassplitter aus der ursprünglichen bzw. nach 5 Tagen umgesetzten Kultur bei gleicher Vergrößerung, 16 Tage alt. Fig. 19: Teil eines fertilen Makrothallus mit pluri- und unilokulären Sporangien, 20 Tage alt. Maßstrecken: Fig. 11 und $13=200 \mu m_{i}$ Fig. 12, 14, 15, $16=50$ $\mu \mathrm{m}$; Fig. 17 und $18=200 \mu \mathrm{m}$; Fig. $19=50 \mu \mathrm{m}$ 
rechte Fäden entspringen (Fig. 18). Die kriechenden Fäden trugen entweder nur einreihige plurilokuläre (Fig. 16) oder auch beiderlei Sporangien auf demselben Thallus (Fig. 15). Nicht anders die Makrothalli: ein Faden aus der 20 Tage alten Kultur trägt unterhalb der schon entleerten plurilokulären die meist noch nicht reifen unilokulären Sporangien (Fig. 19).

\section{DIE “NORMALE” ENTWICKLUNG}

Die mit den Schwärmern aus Fäden des Kulturmaterials in Provasoli-Medium durchgeführten Versuche verliefen wie erwartet. Die großen Schwärmer aus den plurilokulären Sporangien des Makrothallus entwickelten sich entsprechend den Figuren 20 und 21 unmittelbar wieder zu einer Fadengeneration. Wesentlich kleiner sind die Schwärmer aus unilokulären Sporangien; ihre Größe entspricht den pluri-Schwärmern der Mikrothalli (Fig. 22). Aus beiden Arten von Schwärmern entwickelten sich Mikrothalli (Fig. 23). Auf einem Teil von ihnen entstanden schon bald aufrechte Fäden; die Schwärmer einreihiger Sporangien ergaben rasch wieder Mikrothalli. Zusammenfassend läßt sich feststellen, daß alle Kulturen - unabhängig von der Art ihrer Schwärmer - nach kurzer Zeit unter optimalen Bedingungen wieder zu aufrechten Fäden führen.
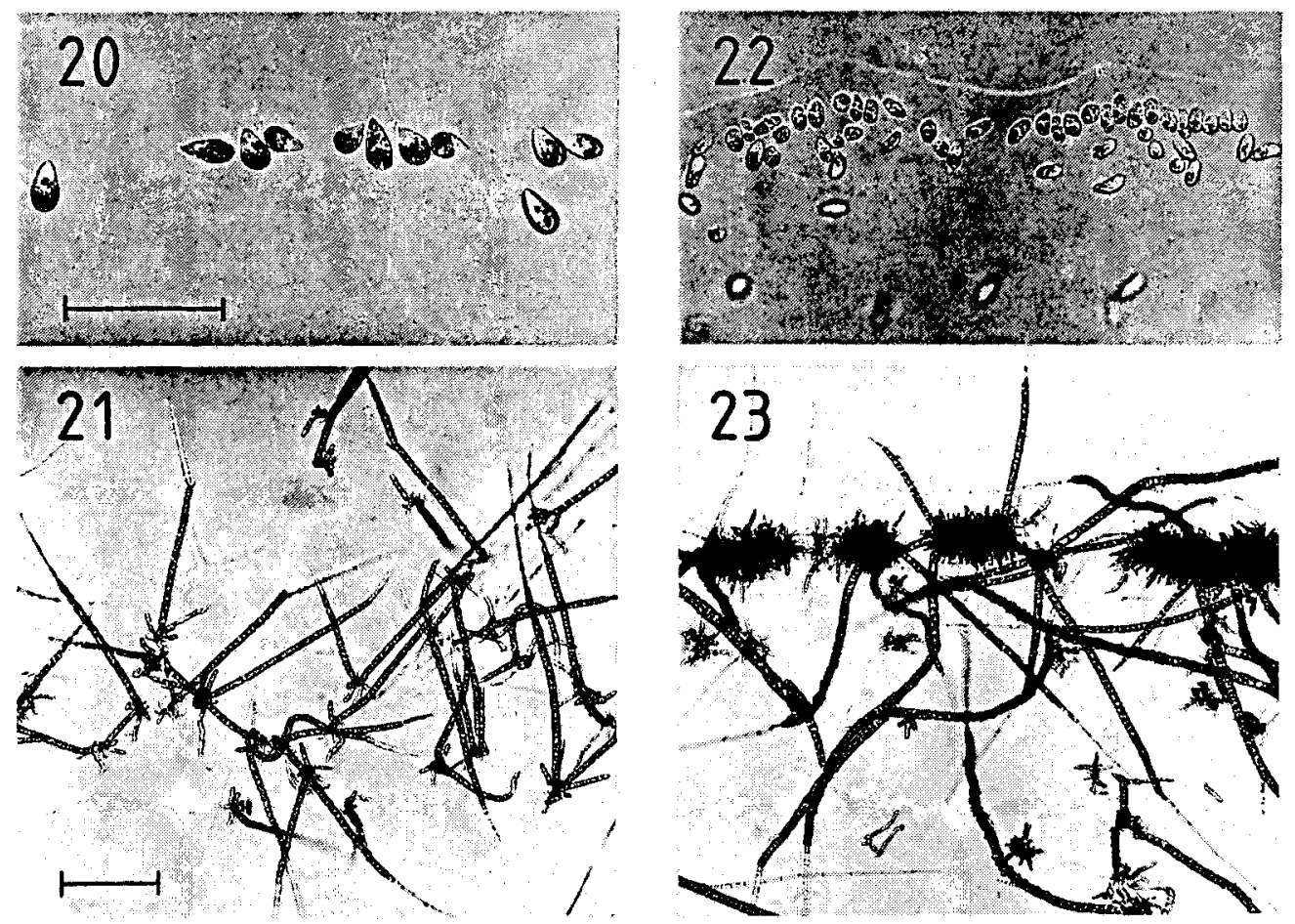

Fig. 20-23. Litosiphon pusillus. Fig. 20 und 21: Schwärmer aus großen plurilokulären Sporangien des Makrothallus und ihre 6 Tage alte Nachkommenschaft. Fig. 22 und 23: Schwärmergemisch aus einer älteren Kultur - wohl meist aus einreihigen Sporangien von Mikrothalli stammend - und ihre 9

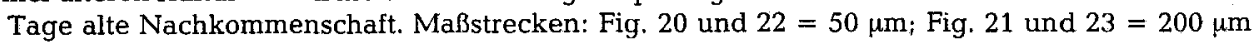




\section{DISKUSSION}

Mikrothalli von Litosiphon sind aus dem natürlichen Milieu nicht bekannt. Wir wissen nicht, ob sie während der Jahreszeit, in der die fädige Pflanze voll entwickelt ist, überhaupt vorkommen. Wenn schon in den Kulturen nach kurzer Zeit aus pluri- und aus uni-Schwärmern wieder fädige Pflanzen entstehen, so ist dies um so mehr unter den natürlichen Gegebenheiten wahrscheinlich, zumal die Mikrothalli nicht obligat in den Lebenszyklus eingeschaltet sind. Daß die Wintermonate als Mikrothalli überbrückt werden, haben schon Sauvageaus Beobachtungen erwiesen. In unseren Kulturen blieben sie als äußerst reduzierte Stadien unter Mangelbedingungen lange lebensfähig.

Die Gattung Pilocladus wurde erst in dem Nachlaß von Kuckuck (1954) beschrieben, er trennte sie wegen ihrer kurzen aufrechten Assimilationsfäden aus der Gattung Streblonema ab. Der Thallus lebt endophytisch im Gewebe schwammiger Wirtsalgen. Kuckuck nahm drei Arten in die Gattung auf, $P$. volubilis (Crouan) von der französischen Atlantikküste, $P$. thuretii (Sauv.) aus dem Mittelmeer und $P$. danicus (Kylin) aus der Ostsee und Südnorwegen. Die Beschreibungen sind ausgezeichnet von Kuckuck illustriert.
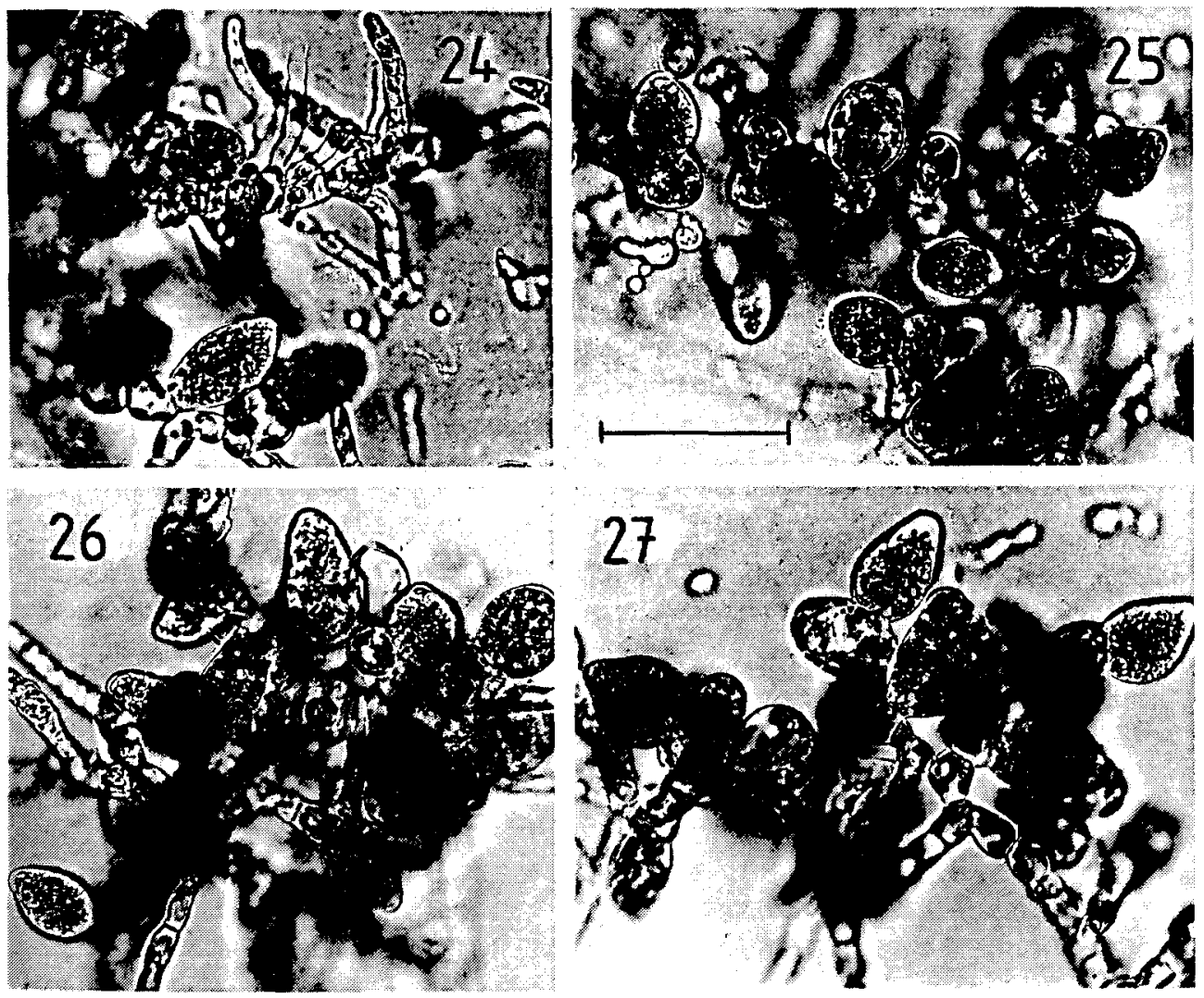

Fig. 24-27. Litosiphon pusillus. Mikrothalli mit unilokulären Sporangien aus verschiedenen Kulturen. Maßstrecke $=50 \mu \mathrm{m}$ 
Trifft die Ansicht von Pedersen zu, daß Litosiphon-Mikrothalli und Pilocladus-Arten identisch seien? Für den Vergleich mit Pilocladus stehen uns nur die in Kulturen erzielten Litosiphon-Mikrothalli zur Verfügung: Pedersens Figuren 15 und 16 sowie die hier an Material aus Helgoland dargestellten. In beiden Fällen fehlt das kennzeichnende Merkmai von Pilocladus, die aufrechten Assimilationsfäden. Wenn auch die Form der langovalen sitzenden unilokulären Sporangien seiner Figur 15 Pedersen zu seiner Hypothese angeregt haben mag, so bleiben sie doch mit höchstens $50 \mu \mathrm{m}$ Länge weit unter der Größe der Pilocladus-Sporangien. In unseren Kulturen sind die reifen unilokulären Sporangien der Litosiphon-Mikrothalli in Form und Größe völlig verschieden von Pilocladus, sie sind rundlich oval bis birnförmig und meist 30-40 $\mu \mathrm{m}$ lang (Fig. 24-27). Damit stimmen sie gut mit Sauvageaus Abbildungen (1933, Fig. 2) überein.

Nach diesen Feststellungen kann die Auffassung von Pedersen nicht bestätigt werden. Er betrachtet alle drei Pilocladus-Arten als synonym mit den Mikrothalli von Litosiphon, obwohl P. thuretii nur aus dem Mittelmeer bekannt ist, wo Litosiphon pusillus nicht vorkommt. Pedersen schließt auch die Möglichkeit der Synonymie von Streblonema oligosporum mit L. pusillus nicht aus. Die Ähnlichkeit dieser Art mit den Mikrothalli von $L$. pusillus reicht für eine solche Annahme nicht aus, nur der experimentelle Nachweis könnte überzeugen.

Nachdem auch South \& Tittley (1986) die Hypothese von Pedersen ohne Vorbehalt übernommen und alle vier "Streblonema"-Arten als Stadien im Lebenszyklus von $L$. pusillus gewertet haben, führten unsere Kulturen zu dem Ergebnis, daß diese Interpretation nicht aufrecht erhalten werden kann.

\section{ZITIERTE LITERATUR}

Dangeard, P., 1965. Sur le cycle évolutif de Litosiphon pusillus (Carm.) Harvey. - Botaniste 49, $47-63$.

Kuckuck, P., 1954. Ectocarpaceen-Studien II. Streblonema. - Helgoländer wiss. Meeresunters. 5 , $103-117$.

Kylin، H., 1933. Über die Entwicklungsgeschichte der Phaeophyceen. - Lunds Univ. Årsskr. (N. F., Avd. 2) 29 (7), 1-102.

Nygren, S., 1975. Life history of some Phaeophyceae from Sweden. - Botanica mar. 18, 131-141.

Nygren, S., 1979. Life histories and chromosome numbers in some Phaeophyceae from Sweden. Botanica mar. 22, 371-373.

Pedersen, P. M., 1984. Studies on primitive brown algae (Fucophyceae). - Opera Bot, 74, 1-76.

Sauvageau, C., 1933. Sur quelques algues Phéosporées de Guéthary (Basses-Pyrénées). - Bull: Stn biol. Arcachon 30, 1-128.

South, G. R. \& Tittley, I, 1986. A checklist and distributional index of the benthic marine algae of the North Atlantic Ocean. Huntsman Marine Laboratory \& British Museum (Natural History), St Andrews \& London, $76 \mathrm{pp}$. 